\title{
ULTRAMETRICALLY INJECTIVE SPACES
}

\author{
JOSÉ M. BAYOD AND J. MARTÍNEZ-MAURICA
}

(Communicated by Dennis Burke)

\begin{abstract}
We study the injective objects in the category of ultrametric spaces with contractive mappings. As a result, the property of spherical completeness is characterized in several new ways related to extensions of uniformly continuous mappings and to extensions of isometries. A construction of the injective envelope is also given.
\end{abstract}

A metric space $Y$ is "injective" if every mapping which increases no distance from a subspace of any metric space $X$ to $Y$ can be extended, increasing no distance, over $X$. In [1], Aronszajn and Panitchpakdi introduced these spaces, calling them "hyperconvex." Isbell, [5], proved that every metric space $X$ has an "injective envelope," i.e., an isometric embedding $e: X \rightarrow E$ such that $E$ is injective and no injective proper subspace of $E$ contains $e(X)$. Recently, Dress [3] introduced the concept of "tight extension" of a metric space $X$ and proved that a "tight span" (i.e., a maximal tight extension) of $X$ is the same as an injective envelope of $X$. Other related results on some interesting specific spaces can be found in [6 and 7].

In this paper we are going to consider the case of ultrametric spaces (i.e., when the metric verifies the strong triangle inequality $d(x, y) \leq \max \{d(x, z), d(z, y)\}$ for every $x, y, z$ in $X)$. Ultrametric spaces used to raise interest only among pure mathematicians; this situation has changed recently, when the concept of ultrametricity appeared in a natural way in several physical modellings of natural phenomena. The interested reader can consult the excellent survey [9] and the references given there, in order to get a feeling of the way and the depth in which ultrametric concepts play a role in some parts of modern physics.

It is easy to prove that no ultrametric space with more than one point is injective (use [11, pp. 46-48]). So we restrict ourselves to the following weaker definition: An ultrametric space $Y$ is said to be ultrametrically injective if every contractive mapping from a subspace of any ultrametric space $X$ to $Y$ can be extended to a contraction over $X$.

Our main results are Theorem 2 (characterizations of ultrametrically injective spaces), Theorem 6 (construction of an ultrametrically injective envelope), and Theorem 7 (existence and equivalence of other ultrametrically injective envelopes). In Theorem 2 we prove that ultrametric injectivity and spherical completeness are the same for ultrametric spaces.

Received by the editors November 19, 1985 and, in revised form, May 5, 1986 and August 15, 1986.

1980 Mathematics Subject Classification (1985 Revision). Primary 54E40.

Key words and phrases. Injective space, ultrametric (space), spherical completeness, tight extension. 
The key result in this paper is our Theorem 1, where we give some useful geometric characterizations of the concept of "ultrametrically tight extension." For that we use the idea of "bases" of metric spaces as defined by Murphy in [8].

\section{Ultrametrically tight extensions.}

DEFINITION. Let $(Y, d)$ be an ultrametric space and $X \subset Y$. We say that $Y$ is an ultrametrically tight extension of $X$ if for every map $d^{\prime}: Y \times Y \rightarrow R$, the following properties imply $d^{\prime}=d$ over $Y \times Y$ :

$(\mathrm{T}-1) d^{\prime}(x, y)=d^{\prime}(y, x) \geq 0$ for every $x, y \in Y$,

(T-2) $d^{\prime}(x, y) \leq \max \left\{d^{\prime}(x, z), d^{\prime}(y, z)\right\}$ for every $x, y, z \in Y$,

(T-3) $d^{\prime}\left(y, y^{\prime}\right) \leq d\left(y, y^{\prime}\right)$ for every $y, y^{\prime} \in Y$,

$(\mathrm{T}-4) d^{\prime}\left(x, x^{\prime}\right)=d\left(x, x^{\prime}\right)$ for every $x, x^{\prime} \in X$.

Following [8] we say that a subset $X$ of a metric space $(Y, d)$ is a basis of $Y$ if $d(x, z)=d(x, y)$ for every $x \in X$ implies $z=y$.

Now let $X$ be closed in the metric space $(Y, d)$. We say that $Y$ is an immediate extension of $X$ if $d(y, X)<d(y, x)$ for every $x \in X$ and $y \in Y \backslash X$.

THEOREM 1. Let $(Y, d)$ be an ultrametric space and let $X$ be a closed subspace of $Y$. Then, the following properties are equivalent:

(1) $Y$ is an ultrametrically tight extension of $X$.

(2) $X$ is a basis of $Y$ and $Y$ is an immediate extension of $X$.

(3) $B[y, d(y, X)]=\{y\}$ for every $y \in Y \backslash X$ (where $B[y, d(y, X)]$ is the closed ball of center $y$ and radius $d(y, X))$.

ProOF. (1) $\Rightarrow(2)$. First we prove that $X$ is a basis of $Y$. Assume that $y, z \in Y \backslash X$ and $d(y, x)=d(z, x)$ for all $x \in X$.

Define $d^{\prime}: Y \times Y \rightarrow R$ by $d^{\prime}(u, v)=0$ if $u, v \in T$, where

$$
T=\{w \in Y: d(w, y) \leq d(w, X)=d(y, X)\},
$$

and $d^{\prime}(u, v)=a(u, v)$ otherwise. Then $z \in T$, and

$$
w, w^{\prime} \in T, \text { and } u \notin T \Rightarrow d(w, u)=d\left(w^{\prime}, u\right) .
$$

Hence, properties (T-1)-(T-4) hold for $d^{\prime}$, therefore $d^{\prime}=d$, and since $d^{\prime}(y, z)=0$, the equality $y=z$ follows.

To prove that $Y$ is an immediate extension of $X$, assume there are $x_{0} \in X$ and $y_{0} \in Y \backslash X$ such that $d\left(y_{0}, X\right)=d\left(y_{0}, x_{0}\right)$. Define $d^{\prime \prime}: Y \times Y \rightarrow R$ by $d^{\prime \prime}(u, v)=$ $d(u, v)$ if $u \neq y_{0} \neq v, d^{\prime \prime}\left(y_{0}, v\right)=d^{\prime \prime}\left(v, y_{0}\right)=d\left(x_{0}, v\right)$ if $v \neq y_{0}$, and $d^{\prime \prime}\left(y_{0}, y_{0}\right)=0$.

Then $d^{\prime \prime}$ trivially satisfies (T-1)-(T-4) but $d^{\prime \prime} \neq d$.

$(2) \Rightarrow(3)$. Suppose that for some $y \in Y \backslash X$ there exists a $y_{0} \in Y \backslash X$ such that $y_{0} \neq y$ and $y_{0} \in B[y, d(y, X)]$. Take $x \in X$ verifying $d\left(y_{0}, x\right) \neq d(y, x)$. If $d\left(y_{0}, x\right)<d(y, x)$ (the other case is similar) we have $d\left(y_{0}, y\right)=d(y, x)>d(y, X)$, which is a contradiction.

(3) $\Rightarrow(1)$. Let $d^{\prime}: Y \times Y \rightarrow R$ be a map satisfying (T-1)-(T-4). We claim that $d^{\prime}(x, y)=d(x, y)$ for all $x, y \in Y$.

There is nothing to prove if $x, y \in X$. First we consider the case of $x \in X$ and $y \in Y \backslash X$. Choose $z \in X$ with $d(z, y)<d(x, y)$. Then $d^{\prime}(z, x)=d(z, x)=d(x, y)$. If $d^{\prime}(x, y)<d(x, y)$, we have $d(z, y)<d(x, y)=d^{\prime}(z, x)=d^{\prime}(z, y)$, which is a contradiction. Finally let $x, y \in Y \backslash X$; since $d(y, X)<d(y, x)$, there is a $z \in X$ 
such that $d^{\prime}(y, z)=d(y, z)<d(x, y)$. Hence, $d(x, y)=d(x, z)=d^{\prime}(x, z)$ and it follows that $d^{\prime}(x, y)=d^{\prime}(x, z)=d(x, y)$ which finishes the proof.

REMARKS. 1. If $Y$ is a tight extension of an arbitrary (i.e., ultrametric or not) space $X$, then $X$ is also a basis of $Y$ (use Theorem 1 of [3]).

2. If $X$ is a compact ultrametric space, there are no proper ultrametrically tight extensions of $X$. This differs considerably from the case of metric spaces (see Theorem 4 of [3]). Also, if $Y$ is a proper tight extension of a compact (arbitrary) metric space $X$, then $Y$ is never an immediate extension of $X$.

An ultrametric space is said to be spherically complete if every collection of closed balls with the binary intersection property has nonempty intersection.

THEOREM 2 . Let $X$ be a complete ultrametric space. Then the following properties are equivalent:

(1) $X$ is spherically complete.

(2) Every $X$-valued uniformly continuous mapping from a subspace of any ultrametric space $Z$ can be extended, with the same modulus of continuity, to $Z$.

(3) $X$ is ultrametrically injective.

(4) Every isometric embedding $T: Y \rightarrow X$ can be extended to an isometric embedding $\bar{T}: Z \rightarrow X$ where $Z$ is any ultrametrically tight extension of $Y$.

(5) $X$ has no proper ultrametrically tight extension.

(6) $X$ has no proper immediate extension.

PROOF. (1) $\Rightarrow(2)$. Let $T: Y \rightarrow X$ be a uniformly continuous mapping with $Y \subset Z$ and consider the modulus of continuity of $T$, i.e., the map $\delta_{T}:(0,+\infty) \rightarrow$ $(0,+\infty)$ defined by

$$
\delta_{T}(\varepsilon)=\sup \left\{d\left(T y, T y^{\prime}\right): y, y^{\prime} \in Y, d\left(y, y^{\prime}\right) \leq \varepsilon\right\} .
$$

Let $\mathfrak{E}$ be the set of all possible $X$-valued uniformly continuous maps $S$ defined on a subspace of $Z$ which contains $Y$ and satisfy: (a) $\left.S\right|_{Y}=T$ and (b) $\delta_{S}=\delta_{T}$.

For the usual ordering, $\mathfrak{E}$ is an inductive set. Let $\bar{T}: \bar{Y} \rightarrow X$ be a maximal element of $\mathfrak{E}$. We only need to prove that $\bar{Y}=Z$. Otherwise, take $z \in Z \backslash \bar{Y}$ and consider the collection of balls in $X$,

$$
B\left[\bar{T} y, \delta_{T}(d(z, y))\right], \quad y \in \bar{Y} .
$$

For any $y, y^{\prime} \in \bar{Y}$ we have

$$
\begin{aligned}
d\left(\bar{T} y, \bar{T} y^{\prime}\right) & \leq \delta_{T}\left(d\left(y, y^{\prime}\right)\right) \leq \delta_{T}\left(\max \left\{d(y, z), d\left(y^{\prime}, z\right)\right\}\right) \\
& =\max \left\{\delta_{T}(d(y, z)), \delta_{T}\left(d\left(y^{\prime}, z\right)\right)\right\}
\end{aligned}
$$

So, by the spherical completeness of $X$, we can take an $x \in \bigcap B\left[\bar{T} y, \delta_{T}(d(z, y))\right]$. Now we extend $\bar{T}$ to $T^{\prime}: \bar{Y} \cup\{z\} \rightarrow X$ by defining $T^{\prime} z=x$.

Then $T^{\prime} \in \mathcal{E}$, and this contradicts the maximality of $\bar{T}$.

$(2) \Rightarrow(3),(3) \Rightarrow(4)$, and $(4) \Rightarrow(5)$ are obvious.

$(5) \Rightarrow(6)$. If $Y$ is a proper immediate extension of $X$, take $y \in Y \backslash X$. By Theorem 1, $X \cup\{y\}$ is a proper ultrametrically tight extension of $X$.

$(6) \Rightarrow(1)$. Assume $X$ is not spherically complete, and let $B\left[x_{\alpha}, r_{\alpha}\right](\alpha \in A)$ be a system of closed balls in $X$ with $B\left[x_{\alpha}, r_{\alpha}\right] \cap B\left[x_{\beta}, r_{\beta}\right] \neq \varnothing$ and thus $B\left[x_{\alpha}, r_{\alpha}\right] \subset$ $B\left[x_{\beta}, r_{\beta}\right]$ or $B\left[x_{\beta}, r_{\beta}\right] \subset B\left[x_{\alpha}, r_{\alpha}\right]$ for all $\alpha, \beta \in A$, but $\bigcap B\left[x_{\alpha}, r_{\alpha}\right]=\varnothing$. Note that for $x \in X \backslash\left(B\left[x_{\alpha}, r_{\alpha}\right] \cup B\left[x_{\beta}, r_{\beta}\right]\right)$ one has $d\left(x, x_{\alpha}\right)=d\left(x, x_{\beta}\right)$. Hence we may 
define an immediate extension $Y=X \cup\{y\}$ of $X$ by choosing for each $x \in X$ some $\alpha \in A$ with $x \notin B\left[x_{\alpha}, r_{\alpha}\right]$ which exists in view of $\bigcap B\left[x_{\alpha}, r_{\alpha}\right]=\varnothing$, and then putting $d(y, x)=d\left(x_{\alpha}, x\right)$.

REMARK. In the category of ultrametric spaces with the uniformly continuous maps as morphisms, Ellis has proved (see [4, Corollary 3]) that the injective objects are exactly the complete spaces.

2. Ultrametrically injective envelopes. Following [5], we call a mapping between ultrametric spaces $e: X \rightarrow E$ an ultrametrically injective envelope of $X$ or a spherical completion of $X$ if $E$ is spherically complete, $e$ is an isometric embedding, and no spherically complete proper subspace of $E$ contains $e(X)$.

Next, we proceed to construct an ultrametrically injective envelope for any given ultrametric space $X$. This direct construction follows the spirit of similar ideas used by Dress in the context of arbitrary metric spaces (cf. Theorems 2 and 3 of $[3]$ ).

DEFINITION. Call

$$
\begin{gathered}
U P_{X}=\{f: X \rightarrow R \mid \text { for all } x, y \in X, d(x, y) \leq \max \{f(x), f(y)\}\} \\
U T_{X}=\left\{f \in U P_{X} \mid \text { for all } x \in X, f(x)=\sup \{d(x, y): f(y)<d(x, y)\}\right\}
\end{gathered}
$$

(where the last sup is understood to be zero in case $f(y) \geq d(x, y)$ for every $y$ ).

LEMMA 3. (a) For $z \in X$, the map $f_{z}: X \rightarrow R, f_{z}(x)=d(x, z)$, is always in $U T_{X}$

(b) The members of $U T_{X}$ are exactly the minimal elements of $U P_{X}$ with respect to the pointwise ordering $\leq$.

Proof. To show that a minimal member of $U P_{X}$, say $f$, is in $U T_{X}$, assume that it is not, define

$$
g(x)=\sup \{d(x, y): f(y)<d(x, y)\},
$$

and prove that $g \in U P_{X}$ (deal separately with the points where $f(y) \geq d(x, y)$ for all $y$ in $X$ ).

The rest of the proof is easy.

LEMMA 4. (a) If $f \in U P_{X}$ and $x, y \in X$, the map $h: X \rightarrow R$ given by

$$
h(z)=f(z) \quad \text { if } z \neq x, \quad h(x)=\max \{d(x, y), f(y)\}
$$

is also in $U P_{X}$.

(b) For $f \in U T_{X}$ and $x, y \in X$ one has $f(x) \leq \max \{d(x, y), f(y)\}$ and therefore one has either $f(x)=f(y) \geq d(x, y)$ or $f(x)=d(x, y)>f(y)$ or $f(y)=d(x, y)>$ $f(x)$.

ProOF. (b) follows easily from (a). As for (a), we have to show that

$$
d(x, z) \leq \max \{d(x, y), f(y), f(z)\}
$$

for all $z \neq x$. But otherwise $d(x, y)<d(x, z)$ and $d(y, z) \leq \max \{f(y), f(z)\}<$ $d(z, x)$, in contradiction to $d(x, z) \leq \max \{d(x, y), d(y, z)\}$. 
THEOREM 5. If for $f, g \in U T_{X}$ one defines $D(f, f)=0$ and $D(f, g)=$ $\inf \{\max \{f(x), g(x)\}: x \in X\}$ if $f \neq g$, then one has

(a) For $f \in U T_{X}$ and $z \in X, D\left(f, f_{z}\right)=f(z)$.

(b) $D$ is an ultrametric on $U T_{X}$.

(c) The map $i: X \rightarrow U T_{X}, i(z)=f_{z}$, is an isometric embedding of $X$ into $U T_{X}$ which maps $X$ onto $\left\{f \in U T_{X}: f^{-1}(0) \neq \varnothing\right\}$.

Proof. (a) Apply Lemma 4, part (b).

(b) Let $f, g, h \in U T_{X}$, assume

$$
\max \{D(f, h), D(g, h)\}<D(f, g),
$$

and call $c=D(f, g)$.

Then for certain $x, y$ in $X$,

$$
f(x) \leq \max \{f(x), h(x)\}<c \leq \max \{f(x), g(x)\}
$$

and

$$
g(y) \leq \max \{g(y), h(y)\}<c \leq \max \{f(y), g(y)\} .
$$

From this we get $f(x)<c \leq g(x)$ and $g(y)<c \leq f(y)$. So, by Lemma 4, part (b), we get $f(x)<c \leq f(y)=d(x, y)$ in contradiction to $d(x, y) \leq \max \{h(x), h(y)\}<c$.

On the other hand, $D(f, g)=0$ implies that for any $z \in X$,

$$
f(z)=D\left(f, f_{z}\right) \leq \max \left\{\left(D(f, g), D\left(g, f_{z}\right)\right\}=g(z)\right.
$$

as well as, symmetrically, $g(z) \leq f(z)$.

(c) The map $i$ is an isometric embedding, as a consequence of part (a).

Suppose that $f \in U T_{X}$ and $f(z)=0$. Then for all $x \in X$,

$$
x \neq z \Rightarrow f(z)<d(x, z)
$$

then, by definition of $U T_{X}, f(x) \geq d(x, z)=f_{z}(x)$. Since $f$ is minimal, $f=f_{z}$.

THEOREM 6. For any ultrametric space $(X, d),\left(U T_{X}, D\right)$ is an ultrametrically injective envelope of $X$.

ProOF. First, we claim that $U T_{X}$ is an immediate extension of $X$ : if $f$ is in $U T_{X} \backslash X, z$ in $X$, and $D(f, X)=D\left(f, f_{z}\right)$, then $f(z)=\inf \{f(x): x \in X\}$, which is different from 0 by Theorem 5 , part (c). Then the function $h$ defined by

$$
h(x)=f(x) \quad \text { if } x \neq z, \quad h(z)=f(z) / 2
$$

is in $U P_{X}$, against the minimality of $f$.

To show that $U T_{X}$ is spherically complete, consider a nontrivial collection of closed balls $\left\{B\left[f_{\alpha}, r_{\alpha}\right]: \alpha \in A\right\}$ with the binary intersection property, and assume that it has empty intersection.

Then, using similar arguments to the ones in the proof of $(6) \Rightarrow(1)$ of Theorem 2, define a function $f: X \rightarrow R$ by

$$
f(x)=f_{\alpha}(x) \quad \text { if } x \notin B_{\alpha} .
$$

Then $f$ belongs to $U T_{X}$ (prove that it is a minimal member of $U P_{X}$ ) and to every ball $B\left[f_{\alpha}, r_{\alpha}\right]$.

Finally, let $Y$ be any spherically complete space between $X$ and $U T_{X}$. Using the characterization (2) of Theorem 1 , prove that $U T_{X}$ is an ultrametrically tight 
extension of $X$; then $U T_{X}$ is obviously an ultrametrically tight extension of $Y$, so, by Theorem $2, Y=U T_{X}$.

Two ultrametrically injective envelopes of the same space $X, e: X \rightarrow E$ and $f: X \rightarrow F$, are said to be equivalent if they are related by an isometry of $E$ onto $F$ over $X$, i.e., an isometry $g: E \rightarrow F$ with $g \cdot e=f$. The following assertions are standard results in categories with injective envelopes (notice that the existence of a spherically complete space which contains any given ultrametric space is guaranteed by the last theorem and also by some other results in the literature; see, e.g., [2, 10]).

THEOREM 7. Let $X$ be any ultrametric space.

(a) For any two ultrametrically injective envelopes (or spherical completions) $E$ and $F$ of $X$ there exists always a unique isometry of $E$ onto $F$ over $X$.

(b) There exists an ultrametrically injective envelope of $X$ within every spherically complete space which contains $X$.

The authors wish to express their gratitude to the referee, who contributed a number of detailed suggestions for the improvement of this paper.

\section{REFERENCES}

1. N. Aronszajn and P. Panitchpakdi, Extension of uniformly continuous transformations and hyperconvex metric spaces, Pacific J. Math. 6 (1956), 405-439.

2. J. M. Bayod, A spherical completion within the infinitesimal hull of an ultrametric space, Quaestiones Math. 7 (1984), 241-249.

3. A. W. Dress, Trees, tight extensions of metric spaces, and the cohomological dimension of certain groups. A note on combinatorial properties of metric spaces, Adv. in Math. 53 (1984), 321-402.

4. R. Ellis, Extending uniformly continuous pseudoultrametrics and uniform retracts, Proc. Amer. Math. Soc. 30 (1971), 599-602.

5. J. R. Isbell, Six theorems about injective metric spaces, Comment. Math. Helv. 39 (1964), 65-76.

6. _ـ, s admits an injective space, Proc. Amer. Math. Soc. 28 (1971), 259-261.

7. J. Mai and Y. Tang, An injective metrization for collapsible polyhedra, Proc. Amer. Math. Soc. 88 (1983), 333-337.

8. G. P. Murphy, A metric basis characterization of Euclidean space, Pacific J. Math. 60 (1975), 159-163.

9. R. Rammal, G. Toulouse, and M. A. Virasoro, Ultrametricity for physicists, Rev. Modern Phys. 58 (1986), 765-788.

10. W. H. Schikhof, Isometrical embeddings of ultrametric spaces into non-archimedean valued fields, Indag. Math. 46 (1984), 47-50.

11. J. H. Wells and L. R. Williams, Embeddings and extensions in analysis, Springer-Verlag, Berlin and New York, 1975.

Facultad de Ciencias, Av. De los Castros, 39071 Santander, Spain 